\title{
Unresolved Privacy and Ethics Issues Related to Learning Analytics in Higher Education and Academic Librarianship
}

\section{Chad Currier}

Word Count (excluding references): 4489

\begin{abstract}
Learning analytics involve big data collection, analysis processes, and technology that are used in higher education institutes and academic libraries to support student success and perform organizational assessment. Since these processes require the input of personally identifiable student and patron information to be effective, there are major ethical and legal considerations that must be addressed concerning privacy. This article demonstrates that privacy concerns about learning analytics can be mitigated by requiring informed consent from participants, establishing protocols for the collection and management of personally identifiable information, and advocating privacy rights of patrons. By synthesizing and expanding on viewpoints from the literature, this article offers recommendations pertaining to the collection, analysis, and management of patron data that are gathered for the purpose of learning analytics.
\end{abstract}

Keywords: academic libraries; information ethics; higher education; learning analytics; patron privacy

Currier, C. (2021). Unresolved privacy and ethics issues related to learning analytics in higher education and academic librarianship. Emerging Library \& Information Perspectives, 4, 117-142. https://doi.org/10.5206/elip.v4i1.13463 


\section{Introduction}

The development of learning analytics technology and practices in higher education and academic libraries presents enticing opportunities to support student success and perform organizational impact assessments. However, learning analytics also raise serious ethical and legal questions related to the handling of personal and private patron data. This article demonstrates that privacy concerns about the adoption of learning analytics in academic libraries can be mitigated by requiring informed consent from participants, establishing protocols for the collection and management of personally identifiable information, and advocating privacy rights of patrons.

In order to satisfy the above-stated objective, relevant literature was reviewed with the aim to familiarize ourselves with the ideas and efforts of leaders in the field. In addition to scholarly works, grey literature such as frameworks, program documentation, and policy statements that have been produced to support the implementation of learning analytics are consulted as well. In the first section of this article, an overview of learning analytics technology and practices is provided. The second section focuses on the need for informed consent from participants. In the third section, the necessity for protocols in the collection and management of patron data is explored. The fourth and final section concentrates on the professional duty of librarians to advocate privacy rights of patrons. As a result of an expansion on points of view synthesized from the literature, we contribute recommendations that pertain to the collection, analysis, and management of library patron data that are harvested for learning analytics in higher education. 


\section{Overview of Learning Analytics}

Learning analytics is understood as "the measurement, collection, analysis and reporting of data about learners and their contexts, for purposes of understanding and optimising learning and the environments in which it occurs" (Long \& Siemens, 2011, p. 34). Learning analytics technology and practices are developed to help administrators, instructors, and students interpret and respond to learning behaviours. As a result, stakeholders are better positioned to evaluate past choices of students and identify institutional trends, and as Kim et al. (2016) demonstrate, they can even perform predictive analytics to anticipate future challenges. In its most rudimentary form, learning analytics benefit instructors that teach courses in which learning management systems are utilized to facilitate student engagement and discussions. These systems present instructors with visualizations such as charts and dashboards so that they can readily identify detached or "at-risk" students (Jones \& Salo, 2018; Verbert et al., 2013). With this information, instructors can then consider a variety of intervention strategies to help students achieve success.

It is no small task to describe the technology that supports learning analytics since there are vast differences among those systems employed. Oakleaf (2018) declares that "many are 'home grown' by individual higher education institutions, and even more are offered by vendors either as single offerings or suites of learning analytics 'solutions'” (p. 11). Rubel and Jones (2016) concur that "learning analytics is not limited to particular technologies and methods. Rather, it is a technology-enhanced practice that encompasses many different tools and techniques to analyze various types and amounts of student data" (p. 144). Notwithstanding the fact that decisionmakers in 
higher education and academic libraries have a breadth of options from which to choose when adopting learning analytics systems, the goal of every implementation - "to understand and optimize learning and the environment in which it occurs" (Pardo \& Siemens, 2014, p. 443) - remains constant.

To meet this goal, data that are relevant to learning behaviours are collected, analyzed algorithmically, and put to effective use. Techniques associated with big data analytics are employed to harvest and compute data that are generated as a result of these behaviours. According to Picciano (2012), big data "is a generic term that assumes that the information or database system(s) used as the main storage facility is capable of storing large quantities of data longitudinally and down to very specific transactions" (p. 12). Even though Dziuban et al. (2012) describe designing a modest learning analytics system with MySQL, Kumari and Babu (2019) expound that tools such as relational databases are ill-equipped to analyze vast, unstructured, and intricate data sets. Jones and Salo (2018) elucidate that the "Big Data ethos" is:

to develop boundless datasets to be used in ways that are often unanticipated at time of collection. Individuals, organizations, and institutions implementing Big Data initiatives argue that taking an ' $n=$ all' approach and aggregating all the data they can get will optimize their data-driven projects (pp. 305-306).

In simple terms, learning analytics practitioners better their chances of finding "interesting, possibly actionable results" among correlations by aggregating greater amounts of diverse behavioural data (Jones, 2019, p. 417). Furthermore, these data sets are used to train algorithms and identify unexplored connections between student behaviours and success. Pardo and Siemens (2014) make clear that the "technology 
evolves together with the capacity to record the events occurring in a learning environment. "Every interaction and resource accessed can be captured and stored [emphasis added]" (p. 438). The story goes that learning analytics betoken an unlimited potential to understand and assist student success if practitioners input just the right data points.

In higher education, students interact digitally with numerous resources and services. "Digital breadcrumbs", or metadata records of their activities, constitute the data that are harvested for learning analytics. Rubel and Jones (2016) clarify that digital trails consist of records of logins and logouts, clickstreams, time stamps of activities such as accessing resources, and records of engagement such as interactions in forum posts. Siemens (2013) illustrates the granularity of breadcrumbs by introducing into the equation behaviours as innocuous as briefly pausing while reading online. By amassing all this information, learning analytics systems create unique profiles of students that are further refined as they progress throughout their education. The findings of a large-scale empirical study into the use of learning analytics in academic libraries across the United States indicate that the captured data include materials usage tracking and records from research consultations, reference, and instruction interactions with staff (Perry et al., 2018). It is interesting to note that the collected data may be inclusive of student behaviours that are wholly unrelated to study habits, grades, or online engagement. Oakleaf (2018) states that information about co-curricular and extracurricular involvement is harvested as well. This is because engagement in areas such as athletics and student associations not only contribute to health and well-being, but also can influence institutional metrics such as attrition and retention (Oakleaf, 2018). We 
live in a digital era where it is conceivable for data streams to capture any and all forms of behaviour that students exhibit while participating in campus life.

\section{Requiring Informed Consent from Participants}

It may interest privacy advocates that learning analytics occur in higher education without either the knowledge or consent of students. Rubel and Jones (2016) expound that "learning analytics is still an emerging technology and practice, and because it is used primarily by instructors and administrators, students have little awareness about its purpose, its goals, and the data it collects" (p. 154). In their study, Perry et al. (2018) report that $58 \%$ of academic library survey respondents declared that they do not inform patrons that data associated with their behaviours are collected and analyzed. In a recent study involving 100 undergraduate participants from eight higher education institutes in the United States, Jones et al. (2020) learned that none of the students remember consenting to data collection and use. These results contrast with findings from unrelated research that more than $90 \%$ of full-time instructors $(n=502)$ surveyed from colleges and universities in the United States agreed that they felt obligated to protect student privacy (VanScoy et al., 2020). Students and library patrons may feel that behavioural data collection and analyses are an affront to their rights, yet Jones and Salo (2018) show that the Family Educational Rights and Privacy Act (FERPA) in the United States relinquishes institutes from "seeking student consent for data collection, [or] retaining and analyzing student data with impunity because the data is generated as part of the educational process" (p. 306). In their framework designed for ethical implementations of learning analytics, Slade and Prinsloo (2013) recommend 
that higher education institutes obtain informed consent from students to participate in behavioural data collection and analyses.

Given that key stakeholders may be unfamiliar with established learning analytics systems, it seems befitting to review the grey literature of Jisc, a UK-based not-for-profit organization that includes learning analytics as part of its portfolio of digital solutions. The Code of Practice for Learning Analytics (Sclater, 2014), for example, reveals that Jisc and its partner institutes and libraries are legally accountable for the data practices associated with learning analytics as per the General Data Protection Regulation (GDPR) in Europe. Notwithstanding this fact, the GDPR does not require consent for either the collection or use of student behavioural data unless those data comprise special category data such as race and ethnicity, political and spiritual beliefs, or sexuality (Sclater, 2020; Sclater \& Bailey, 2015). For the sake of comparison to federal legislation in Canada, the fair information principles that are enumerated in the Personal Information Protection and Electronic Documents Act (PIPEDA) demand that organizations obtain consent to collect personal and private data. However, since public colleges and universities in Canada do not engage in trade and commerce, these institutes are not subject to PIPEDA. Instead, provincial legislation in areas such as privacy and education govern their activities. Reminiscent of the Freedom of Information and Protection of Privacy Act (FIPPA), legislation in Ontario permits educational institutes to collect, analyze, and store personal and private information such as behavioural data if it is deemed necessary to deliver educational services or other activities (Freedom of Information and Protection of Privacy Act, 1990). 
It is distressing that there is little to no recourse for students to opt out from the collection and use of their behavioural data. Oakleaf (2018) writes that "at many institutions all data is used for analysis, regardless of whether students opt-out or not" (p. 63). According to Perry et al. (2018), more than $25 \%$ of academic library respondents who participate in learning analytics declared that there are no means for students to opt out of data collection practices. Policies that pertain to informed consent and the ability for library patrons to opt out would address not only privacy concerns, but moreover, they would recognize and value the autonomy of students who choose to pursue higher education. Rubel and Jones (2016) propose that interactive dashboards could enable students themselves to manage the specific sources and types of data that are used in learning analytics. Their pioneering recommendation is notable and worth further exploration in environments where neither informed consent nor the option to opt out from the collection of personal and private behavioural data is required.

\section{Establishing Protocols for the Collection and Management of Data}

Learning analytics is by no means out of place in the climate of accountability that permeates higher education and academic librarianship. Several authors (Cox, 2018; Dougherty, 2009; Jones, 2019; White \& Blankenship, 2007) note that libraries must demonstrate their worth in order to justify the support that they receive. Lilburn (2017) writes that this need aligns with neoliberal agendas that not only seek to quantify outputs and improve efficiency, but that neoliberalism "influences and informs decisions concerning the criteria and methods used to describe and assess malleable concepts such as relevance, quality, and excellence" (p. 104). A hallmark of neoliberalism in library service structures is the commodification of patrons and their data (Mathios, 
2019). Even though varied metrics such as in-house materials usage statistics (RoseWise \& Irwin, 2016), circulation statistics (Rose-Wiles et al., 2020), and reference transaction statistics (Beirne \& Aldridge, 2012; Dubnjakovic, 2012; Empey, 2010; LeMire et al., 2016; Ryan, 2008) continue to be used to gauge the value of libraries, these methods are ill-suited to establish with confidence authentic correlations between student success and the utilization of library resources and services. Thorpe et al. (2016) write that "changes in student use of the library, both physically and virtually, mean that gate counts, room use counts, in-house print collection tick marks, and attendance for library instruction sessions are insufficient measures for showing student engagement with the library" (p. 373). If academic libraries are increasingly called upon to prove their relevance within higher education ecosystems, then a need exists to adopt instruments to definitively measure the impact that library resources and services have on student success. Organizations that choose learning analytics to serve this purpose must establish and adhere to clear protocols for the collection and management of patron behavioural data.

Organizational protocols that address the collection, use, and management of patron data for learning analytics should prioritize the security of personally identifiable information. Oakleaf (2018) opines that:

personally identifiable information has value to the individuals who generate it, those who maintain it, and others who may use it. Because anything of value may be misused or misappropriated, it is reasonable to be concerned that bad things could happen if individual-level data is [...] mismanaged (p. 61). 
For academic libraries and their parent institutes to gain insights from learning analytics, fine-grained personal data must be collected and used to establish correlations between learning behaviours and student success. Rubel and Jones (2016) explain that "individual linkage is key for analytics to be most useful to institutions and students. In other words, for learning analytics to be useful for individual students, data must retain unique identifiers" (p. 144). Leaders in library organizations that endorse learning analytics may take into consideration both their present and future facility to aggregate sought-after demographic and behavioural data. Michalak and Rysavy (2019), for example, confess that "one of the major reasons we decided to make the move to a new [integrated library system] was for enhanced reporting capabilities. Symphony did not collect the [personally identifiable] data we were looking for" (p. 775). Once libraries determine the breadth of data that they require, and moreover, by what means they should go about collecting it, their focus should shift to the ways in which they will responsibly steward data sets that contain personally identifiable patron information.

Stakeholders must ask key questions of themselves and the systems that they hope to employ before adopting learning analytics. Fortunately, effort has already been committed to discern what sorts of questions demand resolution. For example, the SHEILA Project (2018), an initiative that aims to advance the responsible use of learning analytics in European universities, published a framework that helps institutes to address their specific needs prior to developing plans and policies to actualize learning analytics. This framework not only assists decision makers to strategize ways to satisfy their data-driven goals, but it also guides institutes to conform to current 
GDPR requirements. Similarly, Drachsler and Greller (2016) offer the DELICATE checklist, a matrix that prioritizes trust throughout the design and implementation of learning analytics systems. As a result of such emerging tools, higher education institutes and academic libraries are formulating forward-thinking policies for the responsible adoption of learning analytics.

Ethical and legal questions about the collection and custodianship of student data form the basis for discussions that are advanced by vendors of learning analytics products as well. The abridged Jisc Code of Practice for Learning Analytics, for example, focuses on key principles that address nothing but the ethics and legality of learning analytics: responsibility; transparency and consent; privacy; validity; access; enabling positive interventions; minimising adverse impacts; and stewardship of data (Sclater \& Bailey, 2015). These same eight facets are reiterated in the Jisc Model Institutional Learning Analytics Policy (Sclater, 2016a), an advisory document that is further complemented by the user-oriented Jisc Model Student Guide to Learning Analytics (Sclater, 2016b). Finally, the Jisc Senior Managers' Guide to Learning Analytics (Sclater, 2020) conceptualizes learning analytics in operational ways for decision makers in organizations. For instance, this latter document details how Jisc collects data from student records systems and virtual learning environments, but it stresses that institutions maintain control of their data. Furthermore, Jisc asserts that the systems and processes that it supports not only conform to ethical standards, but moreover, that they comply with the most robust data protection measures that are required by law: "Being clear about which data sources are being used, what they will be used for and who will have access to them is not only good practice but is also 
required under the GDPR" (p. 13). By converging best practices that are proposed by thought leaders in the field, and by ensuring strict compliance with legislation and directives that pertain to data protection, higher education institutes and academic libraries can demonstrate that they prioritize the establishment of transparency and trust in programs that are designed to meet their data-driven needs. As a result, patrons can remain confident that their personally identifiable information and behavioural data will not be exposed to risk.

\section{Advocating Privacy Rights of Patrons}

A significant consideration to address concerning learning analytics in academic libraries is that some of the most pertinent information about patrons, that is, their distinctive reading habits and research behaviours, is not readily accessible in library systems. In general, librarians are unwilling to capture fine-grained behavioural data even though links can indeed be formed between digital resource usage data and unique student-level (Holzmann, 2020) and department-level (Yu \& Shieh, 2014) identifiers by mining and analyzing data from proxy server logs. Paradoxically, this information is collected, managed, analyzed, and purportedly owned by the third-party aggregators and vendors from whom libraries license content. Lynch (2019) writes that: platform providers know a tremendous amount of information about what is being read, and the patterns of reading. Particularly to the extent that they can associate this information with who is doing the reading, they have frighteningly detailed data (p. 9, emphasis in original).

According to Jones and LeClere (2018), vendor conduct of this sort not only disregards traditional information flows, but moreover, it is a "violation of contextual integrity" if 
libraries are understood as safe places in which intellectual freedom is exercised ( $p$. 362).

Some members of the library cadre are disinclined to let vendors have their way with data pertaining to the information behaviours of patrons. Jones and Salo (2018) propose that librarians advocate privacy rights and "require vendors to disclose behavioral data they gather, how long they keep it, and what they do with it" (p. 316), and Dowling (2017) posits that "vendor policies should be posted online where libraries can link to them and patrons can find them" (p. 35). Clickwrap or clickthrough notices, normally utilized as end-user agreements, may serve the purpose of notifying patrons that their distinctive information seeking behaviours are tracked, stored, and analyzed by electronic resource vendors. On the other hand, mutually beneficial, collaborative efforts between librarians and vendors for the optimal use of patron data could also be pursued. Oakleaf (2018) recognizes that it is inevitable that vendors will "determine how to monetize and/or productize" patron behavioural data, but this same author also suggests libraries pay vendors for the right to use that data in their own learning analytics initiatives (p. 66).

Even if the metrics produced through learning analytics processes are now commonly used to inform assessment and decision-making in higher education, various scholars observe a reluctance on the part of academic libraries to adopt student behavioural data collection and analysis techniques for either their own or broader institutional use (Lynch, 2019; Oakleaf, 2018). Jantti and Heath (2016) comment that, consequently, "the critical act of student engagement with library information resources is omitted from learning analytics by default" (pp. 203-204). Technological and legal 
limitations may be valid explanations for the slow uptake of learning analytics in academic libraries, but a more probable cause is the professional ethics to which librarians adhere. Jones (2019) posits that "practices that collect, aggregate, analyze, and act on data revealing students' intellectual behaviors and interests is antithetical to the library profession's commitments to user privacy and intellectual freedom" (p. 419). These commitments are defined no clearer than through guiding documents that are produced by organizations such as the Canadian Federation of Library Associations (CFLA) and the American Library Association (ALA).

In the "Code of Ethics" (CFLA, 2018), a set of guidelines that was adopted from the International Federation of Library Associations and Institutions (IFLA), librarians are led to take up absolute positions in defence of privacy: "The relationship between the library and the user is one of confidentiality and librarians and other information workers will take appropriate measures to ensure that user data is not shared beyond the original transaction" (p. 2). Notwithstanding a level of ambiguity that could be construed in applications of "appropriate measures", librarians advocating patron privacy in their dealings with vendors would certainly keep within the spirit of the guidelines. The professional commitment of librarians for safeguarding confidentiality about patron information behaviours is explicit in the "Library Bill of Rights" (ALA, 1996), and yet the question remains - why do some academic libraries implement systems to collect and analyze student behavioural data? One respondent in Jones (2019) retorts that they "need to put the priorities of the institution ahead of the priorities of ALA" (p. 416). The ALA provides clarification to its stance on privacy in interpretive documents that specifically address the collection and use of patron data by vendors. For example, in 
policy such as "Privacy: An Interpretation of the Library Bill of Rights" (ALA, 2019), libraries are not only urged to negotiate with vendors for the retention of user data, but moreover, they are encouraged to advocate vendor compliance with the NISO Consensus Principles on Users' Digital Privacy in Library, Publisher, and SoftwareProvider Systems (NISO, 2015), a document that expressly addresses the need for organizations to weigh potential benefits against harms when collecting and analyzing patron data.

Opponents of learning analytics may argue that the collection and analysis of student and patron data transgresses research ethics protocols, and that learning analytics should be held to the same standards in academia as any other behavioural study. Multiple researchers recognize a discrepancy between learning analytics practices on the one hand, and research ethics and review board requirements on the other (Drachsler \& Greller, 2016; Jones, 2019; Jones et al., 2020). Pardo and Siemens (2014) in particular bring to light concerns about intersections of the two: "Although educational institutions typically have human research ethics committees or institutional review boards that already process the requests to carry research initiatives involving the collection of personal data, learning analytics poses some new boundary conditions" (p. 442). Ethical quandaries emerge if review boards are not informed of the potential risks associated with big data practices in learning analytics. Interviewees in Jones (2019) relate that learning analytics are "often exempted from more thorough IRB review because the work [is] considered common educational research done to improve teaching and learning methods and tools" (p. 417). A need exists to distinguish between 
instances of researcher-led learning analytics projects and institute-wide operations. Drachsler and Greller (2016) clarify that:

ethical approval is typically only required for "experiments". If a system is rolled out directly and becomes part of the operational infrastructure of an institution, ethical approval is not always sought. The rollout of the new system then has to comply with the privacy and data protection laws according to national legislation (p. 92).

Institutional governance should demand reviews to safeguard the privacy of participants for the reason that these bodies have an ethical responsibility to minimize potential harms. However, by operationalizing the big data processes that are necessary to perform learning analytics in higher education institutes and academic libraries, decision makers and key stakeholders are accountable to legal authorities beyond the campus.

\section{Recommendations}

Learning analytics presents new challenges and opportunities for higher education institutes and academic libraries alike. By utilizing big data techniques, administrators and librarians can not only gain insights into student behaviours, but they can identify correlations between those habits and student success. However, since learning analytics impose privacy concerns about the collection, management, and use of personally identifiable data and unique information-seeking behaviours, the following recommendations are offered for decision makers to consider when strategizing for the adoption of learning analytics in their organizations. 
First, higher education institutes and academic libraries that adopt learning analytics should inform students and patrons about the types of data that are collected as well as the ways that their data will be put to use (ALA, 2019; NISO, 2015; Oakleaf, 2018; Slade \& Prinsloo, 2013). This information could be displayed nearby computer workstations or on terminal screens in languages that are relevant to the local community of users. Libraries should then elicit informed consent from patrons prior to collecting behavioural data (ALA, 2019; NISO, 2015; Slade \& Prinsloo, 2013). Not only should libraries document patron consent, but more importantly, patrons should be empowered to opt out of data collection and analysis if they choose (ALA, 2019; NISO, 2015; Slade \& Prinsloo, 2013).

Second, care should be taken by higher education institutes and academic libraries to responsibly manage the personally identifiable student and patron data that is necessary for learning analytics to be effective. Specifically, there should be clear protocols that delineate who will have access to the data, as well as how long the data will be kept (Dowling, 2017; NISO, 2015; Pardo \& Siemens, 2014). Furthermore, policies should enforce patron confidentiality whenever it is feasible (ALA, 2019; NISO, 2015), and as such, data should be anonymized if personally identifiable information is not necessary to perform the intended function (Oakleaf, 2018).

Third, librarians should advocate patron privacy rights when dealing with thirdparty vendors and content providers (Dowling, 2017; Jones \& Salo, 2018; Lynch, 2019; NISO, 2015). These entities may include organizations that design the learning analytics systems that are employed, as well as content publishers and vendors that collect user data such as search histories and research behaviours. Advocating privacy 
rights with vendors should be essential to license negotiations in libraries going forward (ALA, 2019). There seems to be little point to either maintaining transparency with students about learning analytics on campus or dedicating efforts to safeguarding personally identifiable patron information if libraries are unwilling to defend the privacy of patrons against misuse from external agents.

\section{Conclusion}

Learning analytics practices and technology enable higher education institutes and academic libraries to explore correlations between student behaviours and student success. These insights not only help stakeholders to support at-risk students, but they are utilized to perform organizational impact assessments. However, the adoption of learning analytics in institutes and libraries raises serious ethical and legal questions about the handling of personal and private student and patron data. This article demonstrated that privacy concerns about the adoption of learning analytics can be mitigated by requiring informed consent from participants, establishing protocols for the collection and management of personally identifiable information, and advocating privacy rights of patrons. By uncovering, synthesizing, and further exploring findings from the literature, this article proposes best practices for collecting, analyzing, and managing patron behavioural data that are gathered for the purpose of learning analytics. 


\section{References}

American Library Association. (1996, January 23). Library Bill of Rights. http://www.ala.org/advocacy/intfreedom/librarybill

American Library Association. (2019, June 24). Privacy: An interpretation of the Library Bill of Rights.

http://www.ala.org/advocacy/intfreedom/librarybill/interpretations/privacy

Beirne, H., \& Aldridge, E. (2012). No more tally marks: Making the most out of archived reference transactions. Kentucky Libraries, 76(3), 22-26.

Canadian Federation of Library Associations. (2018, August 27). Code of ethics. http://cfla-fcab.ca/wp-content/uploads/2019/06/Code-of-ethics.pdf

Cox, J. (2018). Positioning the academic library within the institution: A literature review. New Review of Academic Librarianship, 24(3-4), 217-241. https://doi.org/10.1080/13614533.2018.1466342

Dougherty, R. M. (2009). Assessment + analysis = accountability. College \& Research Libraries, 70(5), 417-418. https://doi.org/10.5860/0700417

Dowling, T. (2017). Paths to protecting patron privacy. International Information \& Library Review, 49(1), 31-36. https://doi.org/10.1080/10572317.2017.1270688

Drachsler, H., \& Greller, W. (2016, April). Privacy and analytics: It's a DELICATE issue a checklist for trusted learning analytics. $L A K$ '16: Proceedings of the Sixth International Conference on Learning Analytics \& Knowledge, 89-98. https://doi.org/10.1145/2883851.2883893 
Dubnjakovic, A. (2012). Electronic resource expenditure and the decline in reference transaction statistics in academic libraries. Journal of Academic Librarianship, 38(2), 94-100. https://doi.org/10.1016/j.acalib.2012.01.001

Dziuban, C., Moskal, P., Cavanagh, T., \& Watts, A. (2012). Analytics that inform the university: Using data you already have. Journal of Asynchronous Learning Networks, 16(3), 21-38. https://doi.org/10.24059/olj.v16i3.276

Empey, H. (2010). Transaction analysis of interactions at the reference desk of a small academic library. Partnership: The Canadian Journal of Library \& Information Practice \& Research, 5(2), 1-17.

Freedom of Information and Protection of Privacy Act, R.S.O. § F-31 (1990). https://www.ontario.ca/laws/statute/90f31

Holzmann, B. (2020, March 9). Library services assessment: Electronic resources impact on student success by configuring, harvesting, and preparing hard data from proxy logs as input for statistical analysis [PowerPoint presentation]. In Electronic Resources \& Libraries 2020 Annual Conference, Austin, TX, United States.

https://www.dropbox.com/sh/mj11b52lglkb0ph/AAAr2PFKjf3Qs7Kin_LCwJPla?dl $=0 \&$ preview $=$ Bob+Holzmann++S09+Assessing+Library+Electronic+Resources+Impact+on+Student+Success+ by+configuring+harvesting+and+preparing+hard+data+from+ezproxy+logs.pdf 
Jantti, M., \& Heath, J. (2016). What role for libraries in learning analytics? Performance Measurement and Metrics, 17(2), 203-210. https://doi.org/10.1108/PMM-042016-0020

Jones, K. M. L. (2019). “Just because you can doesn't mean you should”: Practitioner perceptions of learning analytics ethics. portal: Libraries and the Academy, 19(3), 407-428. https://doi.org/10.1353/pla.2019.0025

Jones, K. M. L., Asher, A., Goben, A., Perry, M. R., Salo, D., Briney, K. A., \& Robertshaw, M. B. (2020). “We're being tracked at all times”: Student perspectives of their privacy in relation to learning analytics in higher education. Journal of the Association for Information Science and Technology, 71(9), 10441059. https://doi.org/10.1002/asi.24358

Jones, K. M. L., \& LeClere, E. (2018). Contextual expectations and emerging informational harms: A primer on academic library participation in learning analytics initiatives. In P. Fernandez \& K. Tilton (Eds.), Applying library values to emerging technology: Decision-making in the age of open access, maker spaces, and the ever-changing library (pp. 357-371). Association of College and Research Libraries.

Jones, K. M. L., \& Salo, D. (2018). Learning analytics and the academic library: Professional ethics commitments at a crossroads. College \& Research Libraries, 79(3), 304-323. https://doi.org/10.5860/crl.79.3.304

Kim, D., Park, Y., Yoon, M., \& Jo, I.-H. (2016). Toward evidence-based learning analytics: Using proxy variables to improve asynchronous online discussion 
environments. Internet and Higher Education, 30, 30-43.

https://doi.org/10.1016/j.iheduc.2016.03.002

Kumari, S., \& Babu, C. N. (2019). Transition from relational database to Big Data and analytics. In M. Ahmed \& A-S. K. Pathan (Eds.), Data Analytics (pp. 131-163). CRC Press. https://doi.org/10.1201/9780429446177-6

LeMire, S., Rutledge, L., \& Brunvand, A. (2016). Taking a fresh look reviewing and classifying reference statistics for data-driven decision making. Reference \& User Services Quarterly, 55(3), 230-238. https://doi.org/10.5860/rusq.55n3.230

Lilburn, J. (2017). Ideology and audit culture: Standardized service quality surveys in academic libraries. portal: Libraries and the Academy, 17(1), 91-110. https://doi.org/10.1353/pla.2017.0006

Long, P., \& Siemens, G. (2011). Penetrating the fog: Analytics in learning and education. Educause Review, September/October, 31-40. https://er.educause.edu/articles/2011/9/penetrating-the-fog-analytics-in-learningand-education

Lynch, C. A. (2019). Reader privacy: The new shape of the threat. Research Library Issues, 297, 7-14. https://doi.org/10.29242/rli.297.2

Mathios, K. (2019, July 31). The commodification of the library patron. Gottesman Libraries, Teachers College Columbia University. https://blog.library.tc.columbia.edu/b/23060-The-Commodification-of-the-LibraryPatron 
Michalak, R., \& Rysavy, M. D. T. (2019). Data privacy and academic libraries: Non-PII, PII, and librarians' reflections (part 2). Journal of Library Administration, 59(7), 768-785. https://doi.org/10.1080/01930826.2019.1649969

National Information Standards Organization. (2015, December 10). NISO consensus principles on users' digital privacy in library, publisher, and software-provider systems (NISO privacy principles) [White paper]. http://www.niso.org/publications/privacy-principles

Oakleaf, M. (2018, November 15). Library integration in institutional learning analytics. Syracuse University. https://library.educause.edu/resources/2018/11/libraryintegration-in-institutional-learning-analytics

Pardo, A., \& Siemens, G. (2014). Ethical and privacy principles for learning analytics. British Journal of Educational Technology, 45(3), 438-450. https://doi.org/10.1111/bjet.12152

Perry, M. R., Briney, K. A., Goben, A., Asher, A., Jones, K. M. L., Robertshaw, M. B., \& Salo, D. (2018, September). SPEC kit 360: Learning analytics. Association of Research Libraries. https://doi.org/10.29242/spec.360

Picciano, A. G. (2012). The evolution of big data and learning analytics in American higher education. Online Learning, 16(3), 9-20. https://doi.org/10.24059/olj.v16i3.267

Rose-Wiles, L. M., \& Irwin, J. P. (2016). An old horse revived?: In-house use of print books at Seton Hall University. Journal of Academic Librarianship, 42(3), 207214. https://doi.org/10.1016/j.acalib.2016.02.012 
Rose-Wiles, L. M., Shea, G., \& Kehnemuyi, K. (2020). Read in or check out: A four-year analysis of circulation and in-house use of print books. Journal of Academic Librarianship, 46(4), 1-13. https://doi.org/10.1016/j.acalib.2020.102157

Rubel, A., \& Jones, K. M. L. (2016). Student privacy in learning analytics: An information ethics perspective. The Information Society, 32(2), 143-159. https://doi.org/10.1080/01972243.2016.1130502

Ryan, S. M. (2008). Reference transactions analysis: The cost-effectiveness of staffing a traditional academic reference desk. Journal of Academic Librarianship, 34(5), 389-399. https://doi.org/10.1016/j.acalib.2008.06.002

Sclater, N. (2014, November). Code of practice for learning analytics: A literature review of the ethical and legal issues. Jisc. https://repository.jisc.ac.uk/5661/1/Learning_Analytics_A-_Literature_Review.pdf

Sclater, N. (2016a). Model institutional learning analytics policy. Jisc. https://analytics.jiscinvolve.org/wp/files/2016/11/Jisc-Model-Institutional-LearningAnalytics-Policy-v0.1.pdf

Sclater, N. (2016b). Model student guide to learning analytics. Jisc. https://analytics.jiscinvolve.org/wp/files/2016/11/Jisc-Model-Student-Guide-toLearning-Analytics-v0.1.pdf

Sclater, N. (2020). Senior manager's guide to learning analytics. Jisc. https://repository.jisc.ac.uk/7782/1/senior-managers-guide-to-learninganalytics.pdf 
Sclater, N., \& Bailey, P. (2015). Code of practice for learning analytics. Jisc. https://repository.jisc.ac.uk/6985/1/Code_of_Practice_for_learning_analytics.pdf

SHEILA Project. (2018). SHEILA framework v.2. https://sheilaproject.eu/sheilaframework/

Siemens, G. (2013). Learning analytics: The emergence of a discipline. American Behavioral Scientist, 57(10), 1380-1400. https://doi.org/10.1177/0002764213498851

Slade, S., \& Prinsloo, P. (2013). Learning analytics: Ethical issues and dilemmas. American Behavioral Scientist, 57(10), 1510-1529. https://doi.org/10.1177/0002764213479366

Thorpe, A., Lukes, R., Bever, D. J., \& He, Y. (2016). The impact of the academic library on student success: Connecting the dots. portal: Libraries and the Academy, 16(2), 373-392. https://doi.org/10.1353/pla.2016.0027

VanScoy, A., Jones, K. M. L., Bright, K., \& Harding, A. (2020). Instructors' understanding of and responses to student privacy in the datafied classroom [Poster presentation]. Proceedings of the Association for Information Science and Technology, 57(1), e400. https://doi.org/10.1002/pra2.400

Verbert, K., Duval, E., Klerkx, J., Govaerts, S., \& Santos, J. L. (2013). Learning analytics dashboard applications. American Behavioral Scientist, 57(10), 15001509. https://doi.org/10.1177/0002764213479363 
White, L. N., \& Blankenship, E. F. (2007). Aligning the assessment process in academic libraries for improved demonstration and reporting of organizational performance. College \& Undergraduate Libraries, 14(3), 107-119. https://doi.org/10.1300/J106v14n03_08

Yu, C.-Y., \& Shieh, J.-C. (2014). The study of analytical model of library electronic resources usage: A case of medical electronic resources. Journal of Educational Media \& Library Sciences, 51(5), 57-89. https://doi.org/10.6120/JoEMLS.2014.51S/0644.RS.AM 